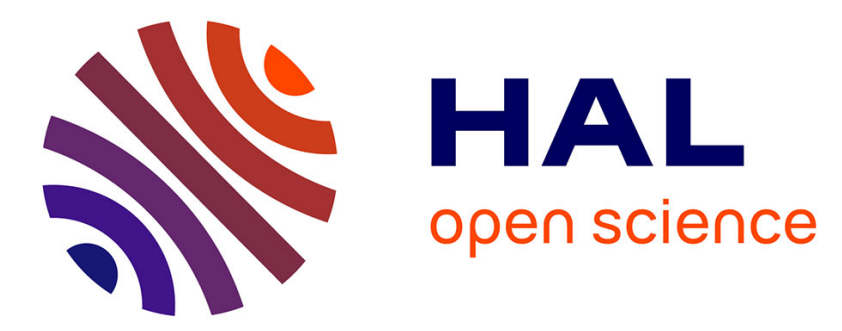

\title{
Les céramiques des Martres-de-Veyre (Allier) et de Chémery (Moselle) au Musée Archéologique de Strasbourg
}

\author{
Jean-Jacques Hatt
}

\section{To cite this version:}

Jean-Jacques Hatt. Les céramiques des Martres-de-Veyre (Allier) et de Chémery (Moselle) au Musée Archéologique de Strasbourg. Gallia - Fouilles et monuments archéologiques en France métropolitaine, 1958, 16 (2), pp.251-261. 10.3406/galia.1958.2232 . hal-01924557

\section{HAL Id: hal-01924557 \\ https://hal.science/hal-01924557}

Submitted on 3 Mar 2020

HAL is a multi-disciplinary open access archive for the deposit and dissemination of scientific research documents, whether they are published or not. The documents may come from teaching and research institutions in France or abroad, or from public or private research centers.
L'archive ouverte pluridisciplinaire HAL, est destinée au dépôt et à la diffusion de documents scientifiques de niveau recherche, publiés ou non, émanant des établissements d'enseignement et de recherche français ou étrangers, des laboratoires publics ou privés.

\section{(1) (1) $\$$}

Distributed under a Creative Commons Attribution - NonCommercial - NoDerivatives| 4.0 
de tenons que l'on voit sous le pied gauche.

Travail délicat. La musculature, les plis de la tunique, le détail des doigts des mains et des pieds sont rendus avec finesse. Traces d'oxydation.

Inédit.

Supplement, 6. --- Anneau d'agrafe (?). Dépôt de fouilles de Bavai, no 7 Z 75 . Trouvé le 25 août 1957 , dans des remblais, vers le sommet de l'angle formé par le côté Ouest du front 0.-N.-O. de l'enceinte et le côté Sud de l'édifice à trois nefs. Longueur 5 centimètres ; largeur $4 \mathrm{~cm} .4$; épaisseur variant de 1 à 5 millimètres (fig. 6).

Fonte pleine. Patine vert sombre. Traces d'oxydation.

L'objet forme un rectangle ajouré, divisé en deux compartiments par une barre verticale. Dans chaque compartiment un ornement en forme de S. D'un côté, dans le prolongement de la barre médiane, le cou et la tête, allongée et plate, d'un animal fantastique. Entre les oreilles, une saillie arrondie, peut-être une corne. Le grand côté du rectangle opposé à la tête porte un anneau, de section rectangulaire, déformé et aminci considérablement vers l'endroit oì frottait l'agrafe. Le plat de l'anneau, de la barre médiane et des grands côtés sont ornés de points en creux.

Inédit. Henri BiÉvelet.

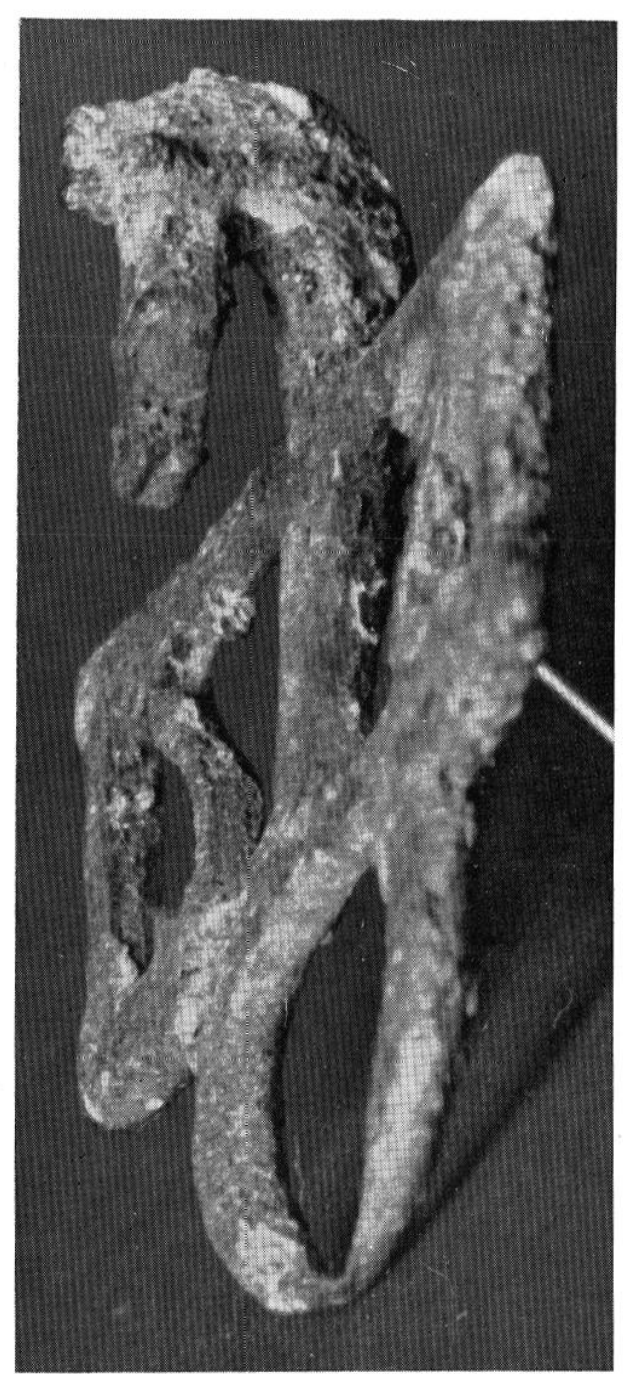

Fig. 6. - Anneau d'agrafe (?), trouvé en 1957.

\section{Les Céramioues des Martres-de-Veyre (Allier) et de Chémery (Mosflle) au Musée Archéologique de Strasbourg}

Les découvertes de Welter et de E. Delort ${ }^{1}$ ont permis de fixer le siège

(1) E. Delort, La céramique de Sallo et de Salurninus, dans. Annuaire de la Société d'His- toire el d'Archéologie de la Lorraine, 1935. Qu'il me soit permis ici de remercier très chaleureusement M. Jan de Groot, spécialiste hollandais très éminent en céramique sigillée, qui m'a prodigué ses directives et ses encouragements, et qui m'a conseillé de rédiger cet article. 
principal de la fabrique de Satto. Ce potier gallo-romain, qui a connu un très grand rayonnement dans la Gaule du Nord-Est, et qui est de ccux dont le style marque une véritable révolution dans le style de la céramique gauloise, était resté longtemps insaisissable. Le récent ouvrage de E. Delort ${ }^{2}$, donnant un inventaire de la céramique découverte à Chémery par Welter, devait révéler qu'à côté de Sallo, avaient travaillé à Chémery un second et un troisième ateliers, les productions du second ayant été jusqu'alors placées à tort sous l'étiquette de l'ollicine de Luxeuil. Le maitre principal de ce second atelier a été considéré par E. Delort comme étant originaire de Lezoux, dont il reproduit certains poinçons ${ }^{3}$. On se doutait depuis longtemps que les officines du Nord-Est avaient été fondées, à la fin du Ier $^{\text {et }}$ au début $d u \cdot \mathrm{x}^{\mathrm{e}}$ siècle, par des maîtres céramistes venus, soit du Midi, soit de la Gaule Centrale. On trouvait à Chémery un important jalon, démontrant cette filiation.

En réalité, il ne semble pas que le second atelier de Chémery soit originaire précisément de Lezoux. Depuis lors, en effet, les recherches et les premières publications de $R$. Terrisse sur l'officine des Martres-de-Veyre (Allier) ont mieux mis en évidence l'importance de cet atelier en ce qui concerne les rapports entre le Centre et le Nord-Est de la Gaule. R. Terrisse a notamment décou-

(2) E. Delort, Les vases ornés de la Moselle, Nancy 1953.

(3) E. Derort, L'atelier de Sallo, vases unis, 3.000 marques, Mémoires de l'Académie nationale de Mctz. CXVIIe année, II e sér., XVII, p. 24 du tirage à part : "j'ai acquis la conviction absolue que l'atelier de Satto était une filiale de L.ezoux "; et In., Vases ornées de la Moselle, p. 191. vert, aux Martres des marques du fameux potier Ranto ${ }^{4}$ qui était auparavant réputé avoir travaillé à Luxeuil ${ }^{5}$ et dont le rôle dans la fondation des ateliers du Nord-Est, s'il n'est pas encore à l'heure actuelle tout à fait précisé, est absolument certain. Ajoutons qu'un ouvrage sur les poliers du Centre de la Gaule d'après les collections anglaises vient de paraître en GrandeBretagne el met l'accent sur l'œuvre des principaux potiers des Martres et sur leurs rapports étroits avec l'ollicine de Chémery ${ }^{6}$.

Indépendamment de ces travaux, j'avais entrepris, depuis 1948, l'inventaire méthodique de la céramique sigillée de Strasbourg. I e classement des réserves du Musée archéologique, auparavant entassées dans une cave obscure et dont la réorganisation a été entreprise par mes soins, devait amener certaines surprises intéressantes. Quelques modestes tessons, ramassés sur le terrain même des Martres-de-Veyre, m'avaient permis, dès 1942, de prendre connaissance de cette céramique, et d'en identifier certains poinçons distinctifs. C'étaient en particulier deux motifs de remplissage bien caractéristiques : une sorte d'ornement en feuillage stylisé, affectant grossièrement la forme d'une ancre (fig. 1, no 1), et, d'autre part, une sorte de chevron, aux côtés fortement incurvés et finement dentelés (fig. 1, no 4).

Je devais retrouver, en 1950 , ces motifs

(4) R. Terriss:, Eine Sigillata Töpferei in Martres-de-Veyre (Frankreich), in Germania, XXII, 1954, p. 171.

(5) E. FöI.zer, Die Bilderschüsseln der osigallischen Sigillata Manufacturen, Bonn, 1913, p. 5, 6, 7.

(6) J. A. Stankiein et Grace Simpson, Central Gaulish Potters, Oxford, 1958 (University of Durham Publications). 


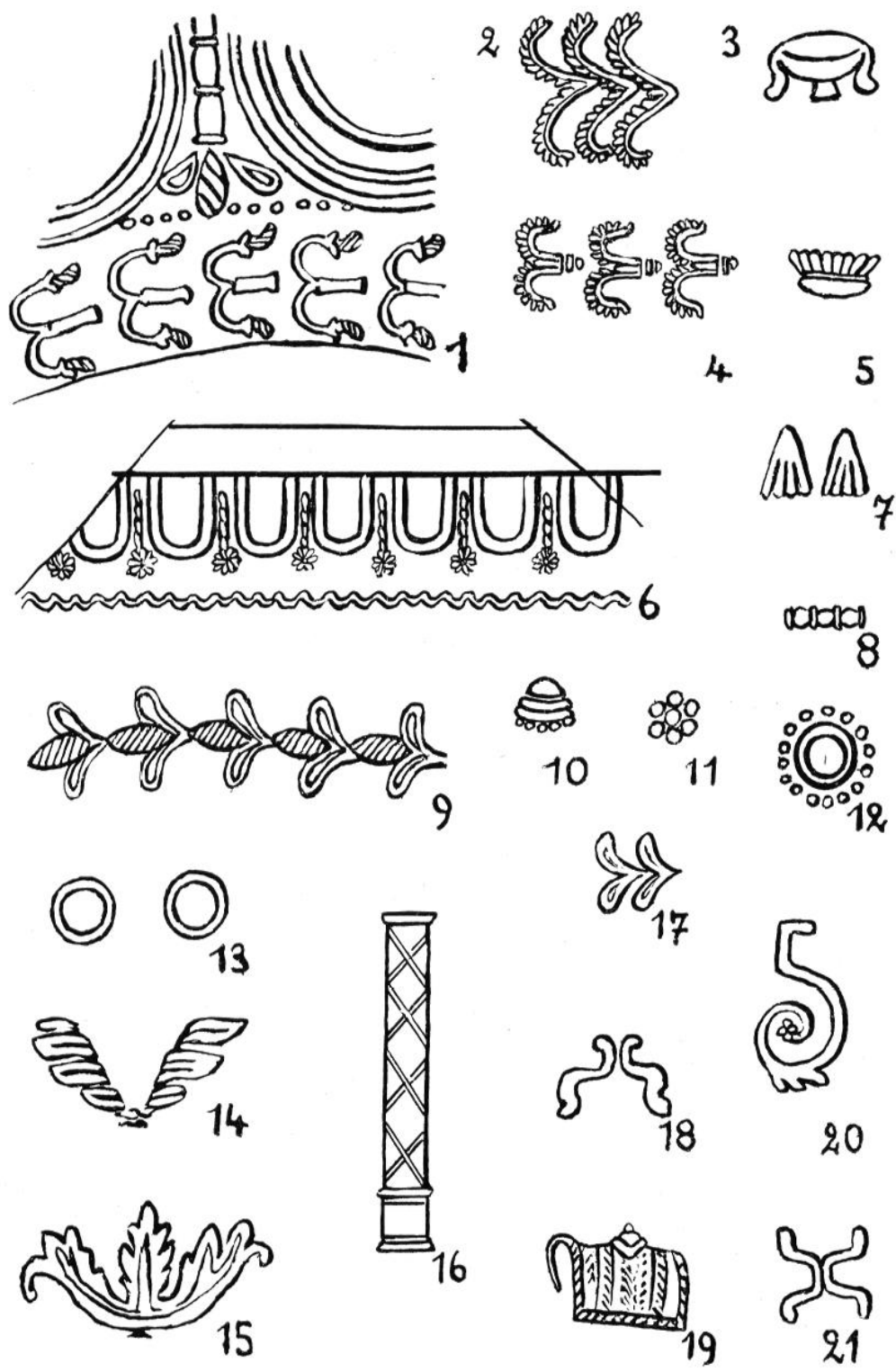

Fig. 1. - Motifs de remplissage des Martres-de-Veyre accompagnant les oves 6 : Potier aux amours et Potier aux gladiateurs. - Dessin C. Sauer,

sur une série de fragments sigillés de très beau style, provenant de Strasbourg et faisant partie des anciennes découvertes faites dans la ville à l'occasion des travaux de tout-à-l'égout. Un certain nombre de ces fragments présentaient, soit en frises continues, soit isolément, la fameuse ancre de feuillage, thème de décoration peu courant et par là même très caractéristique. Certains de ces tessons avaient été, déjà du temps de Forrer, groupés pour reconstituer un vase, de forme Dragendorff 37 (fig. 2). Ce vase, d'une belle technique, compor- 

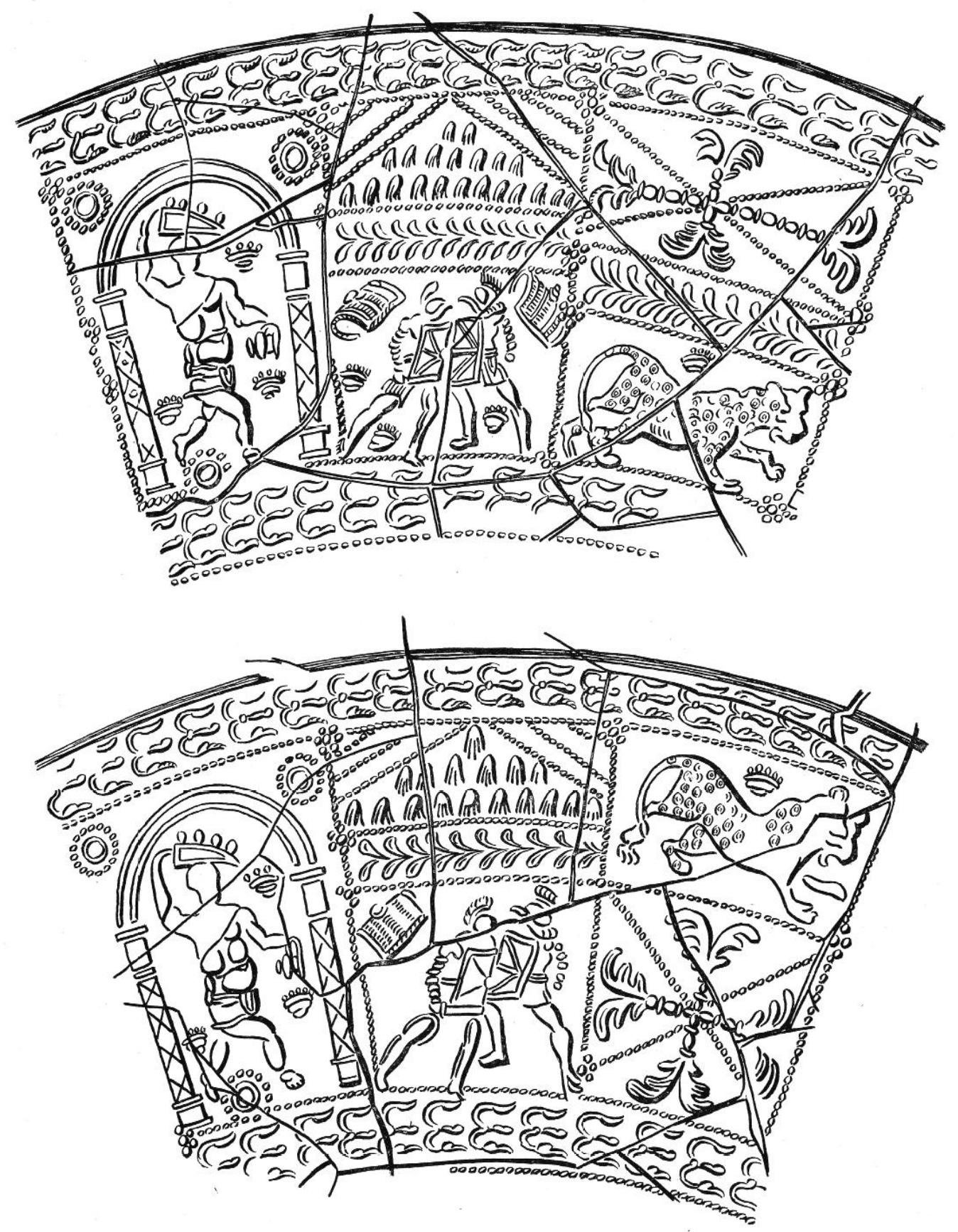

Fig. 2. - Vase des Martres-de-Veyre, au Musée de Strasbourg : Potier aux gladiateurs. Dessin H. Zumslein. 
tait à lui seul un véritable répertoire de motifs, sur la base duquel nous avons pu constituer une première série, comportant des œuvres céramiques de la même officine et du même potier. Un des maîtres céramistes les plus importants des Martres-de-Veyre avait pu ainsi être isolé et identifié parmi les produits de la Gaule du Centre, importés à Argentorate. Nous appellerons ce potier, provisoirement, le "Potier aux gladiateurs", parce que deux de ses motifs caractéristiques sont une paire de gladiateurs et des boucliers de gladiateurs.

Le répertoire de ce potier comprend une ove, de style assez pur et de très bonne technique (fig. 1, no 6). En sélectionnant les fragments sigillés décorés de la même bordure d'oves, nous parvînmes, par rapprochements successifs, à identifier un autre potier de la même officine. Si les productions de ce dernier sont apparentées, par les oves et les molif́s essentiels de remplissage, à celles du Maître aux gladiateurs des Martres, elles sont assez analogues, pour le style et le groupement des figures, à celles de Libertus et de Butrio de Lezoux : même association libre des figures, mêmes modelés anatomiques très étudiés et remarquablement rendus (fig. 3 , nos 1,4 , $5,6,7)$. Nous avons nommé provisoirement ce potier, qui parait avoir travaillé aux Martres-de-Veyre à la fin du $\mathrm{I}^{\text {er }}$ et au début du $\mathrm{II}^{\mathrm{e}}$ siècle, le Maître Potier aux amours, parce qu'il semble avoir une prédilection marquée, dans son répertoire, pour les figures d'Éros ailés.

Ces deux potiers des Martres-de-Veyre une fois identifiés, quelle ne fut pas ma surprise, en feuilletant les planches du livre de E. Delort sur les vases ornés de la Moselle, de constater que le Maître aux gladiateurs des Martres a certainement travaillé à Chémery. Sa production $\mathrm{y}$ est actuellement connue par un moule (Delort, Chémery, p. 184, pl. 80, $n^{\circ} 605$, ici fig. $3, n^{\circ} 2$ ), ainsi que par trois fragments décorés de reliefs (ibid., p. 224, pl. 92, nos 329, 9284, 1936). Le moule, identifié à tort par E. Delort comme ayant été fabriqué à la Madeleine ${ }^{7}$, porte en réalité huit des poinçons les plus caractéristiques du Maître aux gladiateurs des Martres : les gladiateurs du vase de Strasbourg, les pilastres enrubannés (fig. 1, no 16), les deux dauphins (fig. 1, no 18), le petit trépied (fig. 1, no 3 ), les boucliers bouletés (fig. 1, $\mathrm{n}^{\mathrm{os}} 5$ et 10 ), les grands boucliers de gladiateur de forme hémicylindrique (fig. 1, $\mathrm{n}^{\circ} 19$ ), la spirale (fig. 1, no 20).

D'autre part, le maître principal du second atelier de Chémery (l'Anonyme, d'après Delort), ainsi que celui que E. Delort appelle le Troisième Potier, présentent dans leur répertoire un certain nombre d'éléments communs avec le Potier aux gladiateurs, nolamment les boucliers bouletés (fig. 1, no 5), les acanthes ( $\left.n^{\circ} 15\right)$, les abeilles (no 9 ), les grands et petits chevrons dentelés $\left(n^{0 s} 2 \text { et } 4\right)^{8}$. Fncore faut-il dire que c'est le Troisième Potier qui se rapproche le plus des productions des Martres, notamment parce qu'il utilise dans ses frises les motifs nos 2 et 4 des Martres 9 .

(7) E. Delort, Vases ornés de la Moselle, p. 185, no $^{\circ} 537$.

(8) IDEM, ibidem, pl. 79 , p. 182 , moule 1852, type $n^{\circ} 15$ des Martres, voir ici fig. 1; ibid. 1907, type no 14 des Martres; pl. 80, p. 184, moule 8982, motifs 3 et 5 des Martres; pl. 89, p. $215,8,7,36,6956,778,6961$, motif no 2 des Martres utilisé en frise; pl. 90, p. 218, motif no 4 des Martres utilisé en frise sur les fragments $817,136,742,4376,807,105$. Voir également ici fig. 5 et 6 .

(9) Certains des fragments classés par E. Deiont parmi les produits du Troisième Polier : Vases ornés de la Moselle, p. 215, pl. 89, 21, VII, 1936, 778, doivent être placés sous la rubrique du Maître du second atelier (l'Anonyme) dont ils présentent le style et les motifs de remplissage. 

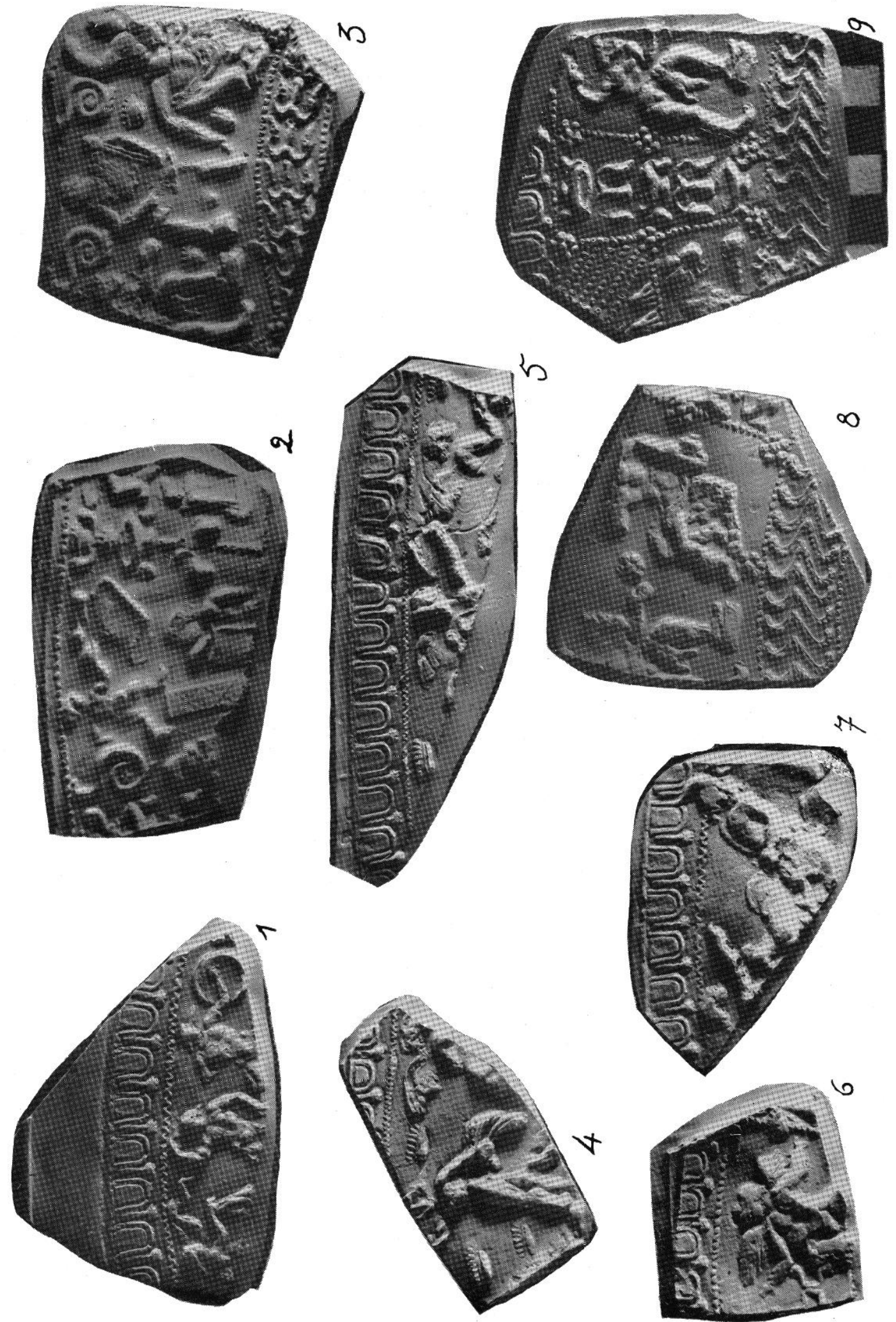

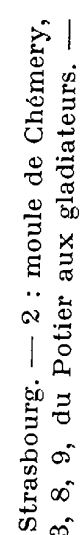

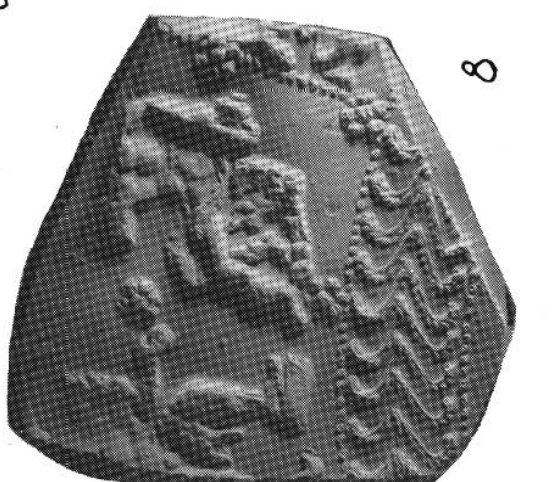

व

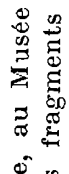

స.

突苑

क्षे

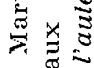

वै : ष

\&

N

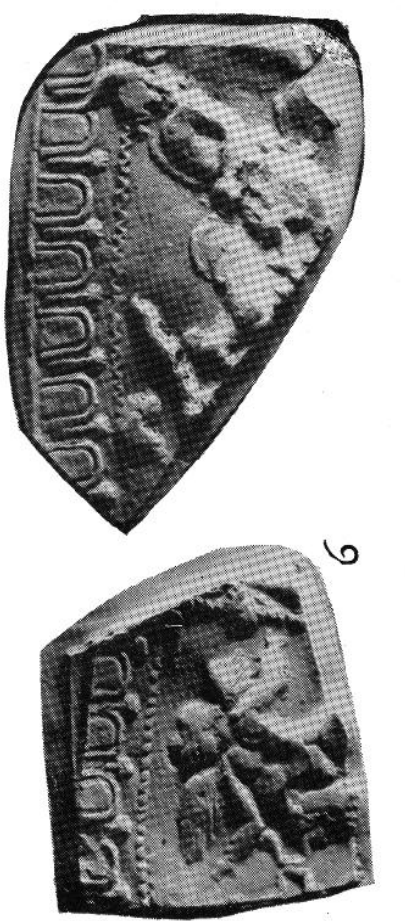

ชั

क्ष

告

की की

需

की

里

绕

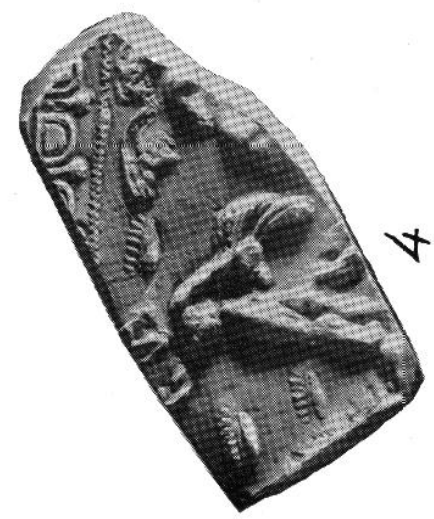

os

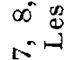

01

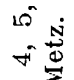

i⿱

1 品

空 


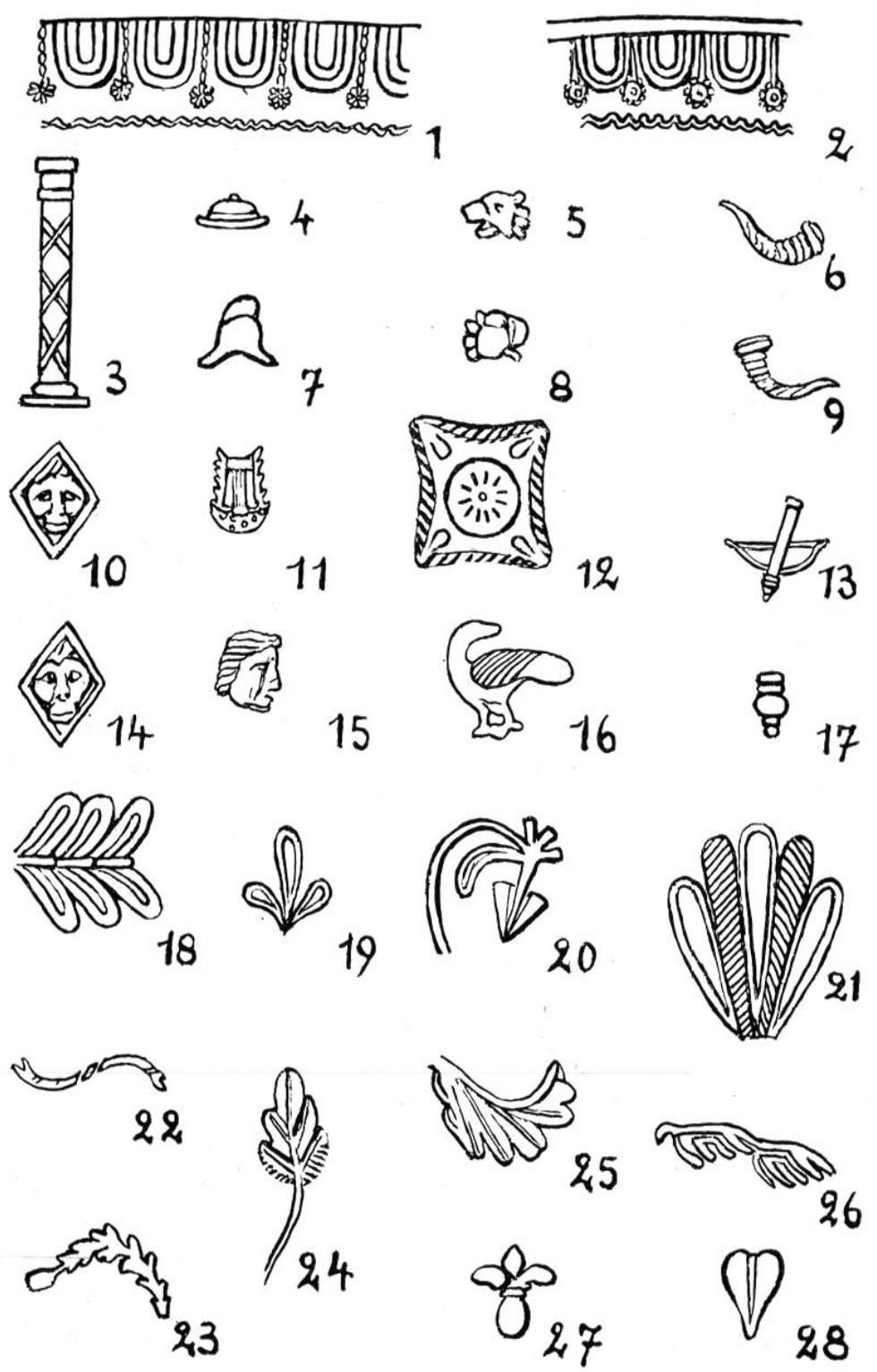

Fig. 4. - Motifs de remplissage du Maître du second atelier de Chémery. - Dessins C. Sauer.

D'ailleurs, son style est très proche de celui de Ranto et Ioenalis ${ }^{10}$, dont les

(10) Comparez notamment avec les fragments de Chémery des pl. 89 et 90 de Delort, les vases signés de Ranto et de Ioenalis: vase de Ranto, signé, trouvé à Heddernheim, dans Mitteilungen über römische Funde in Heddernheim, pl. XXII, fig. XI, ce vase est reproduit par
Oswald et Pryce dans An Introduction to the Study of Terra Sigillata, pl. XXVIII, 12. Autre vase de Heddernheim, non signé, mais sûrement de Ranto, dans KNorn, Die verzierten Terrasigillatagefässe von Rottweil, Stuttgart, 1907, pl. IX, 11 ; motifs nos 4 et 5 des Martres, associés aux motifs nos 20, 17 et 25 de Chémery. ID., ibid., pl. IX, fig. 3 : motif no 22 de Chémery, associé avec le motif $n^{\circ} 4$ des Martres. - Voir encore D. PryGE et E. Birley, The first Roman Occu- 


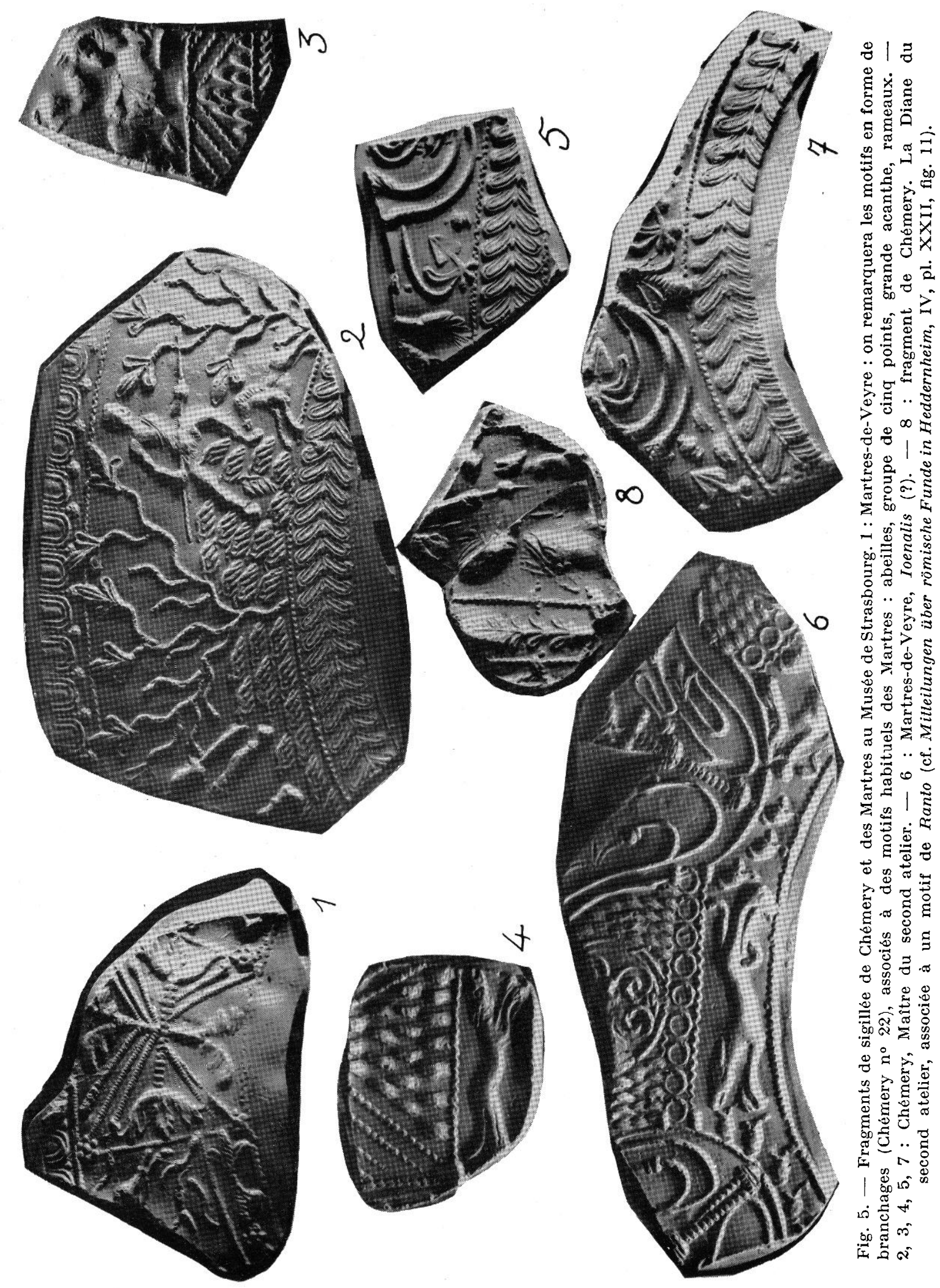


signatures ont été trouvées par R. Terrisse aux Martres-de-Veyre ${ }^{11}$.

Cette enquête prouve qu'il y a eu à Chémery trois maîtres potiers, qui entretenaient avec les officines des Martres-de-Veyre des rapports certains : le premier, peu représenté jusqu'à ce jour à Chémery, n'est autre que le Maître aux gladiateurs des Martres ${ }^{12}$. Le ser.ond, celui que E. Delort appelle le Troisième potier de Chémery, fait partie par son style, aussi bien que par son répertoire, du groupe Ranto-Ioenalis, dont les productions, en partie signées, ont été depuis longtemps identifiées en Allemagne et en Grande-Bretagne ${ }^{13}$. Ce potier a travaillé aux Martres-de-Veyre. Le troisième est celui qui avait été autrefois assimilé à tort à Ranto, et attribué, également à tort, à l'officine de Luxeuil, sous le nom de Maître au bouclier et au casque, par E. Foelzer ${ }^{14}$. C'est celui que E. Delort appelle le Maître du second atelier, ou l'Anonyme de Chémery. Ce maître présente dans son répertoire un certain nombre d'éléments communs avec le Maître aux gladiateurs, et avec le groupe Ranto-Ioenalis mais il parait

pation of Scotland, in Journal of Roman Studies, 1935, pl. XVI : l'association des motifs 11,13 et 17 de Chémery avec les motifs 9 , 4 et 17 des Martres, sous une frise d'oves 1 de Chémery. A lui seul, ce vase tendrait à prouver que le Troisième Potier de Chémery, selon E. Delort, est un maître des Martres, du groupe RantoIoenalis. - Pour les vases signés Ioenalis, voir ibid., pl. XV.

(11) Germania, XXII (1954), 3, p. 172, no8 51 et 68

(12) Très bel exemple de produit du Potier aux gladiateurs, signalé par J. De Groot à Utrecht, voir Volgraff, VAN Hoors, Opgravingen op het Domplein te Utrecht, III, Haarlem, 1936, p. 108, fig. $66, n^{\circ} 47$.

(13) Voir plus haut, n. 10.

(14) Fölzer, Die Bilderschüsseln der ostgallischen Sigillalamanufakturen, Bonn, 1913, p. 4-7 et pl. I, nos 3 à 17. avoir, d'autre part, emprunté également des motifs à Lezoux, comme E. Delort l'avait très bien constaté. Enfin et surtout, il possède une grande variété de très beaux motifs figurés, de qualité égale ou supérieure à celle des meilleurs maîtres, comme Liberlus ou Butrio. Mais il ne paraît pas pouvoir être superposé avec le Maître aux amours des Martres, dont il ne possède aucun des motifs particuliers. C'est donc très certainement un maître qu'il convient d'individualiser en tant que tel, et en le distinguant à la fois du groupe RantoIoenalis des Martres-de-Veyre et de Chémery (Troisième Potier de Delort), du Potier aux gladiateurs, et du Potier aux amours. Ses points de contact avec les potiers connus pour avoir travaillé aux Martres ne doivent pas faire illusion : il a subi leur influence, puisqu'il a travaillé avec eux mais il ne saurait se confondre avec eux. Ce maître, qu'il convient de considérer comme un des meilleurs artistes de la sigillée gauloise, a-t-il quelque temps travaillé aux Martres-de-Veyre, avant de s'installer à Chémery, où il parait avoir exercé la plus grande part de son activité, au cours de la première moitié du II ${ }^{\mathrm{e}}$ siècle?

Pour répondre à cette question, dont l'importance est aisée à comprendre, un examen comparatif des matériaux trouvés aux Martres-de-Veyre et à Chémery sera absolument nécessaire. Cette confrontation exigera, pour être efficace et sûre, un répertoire complet des motifs employés par les divers potiers de l'une et l'autre officines, particulièrement des oves et des motifs de remplissage, conçus sur le modèle que nous donnons ici, ainsi que de bonnes photographies de moulages développés, dans le genre de celles que nous publions dans cet article. 


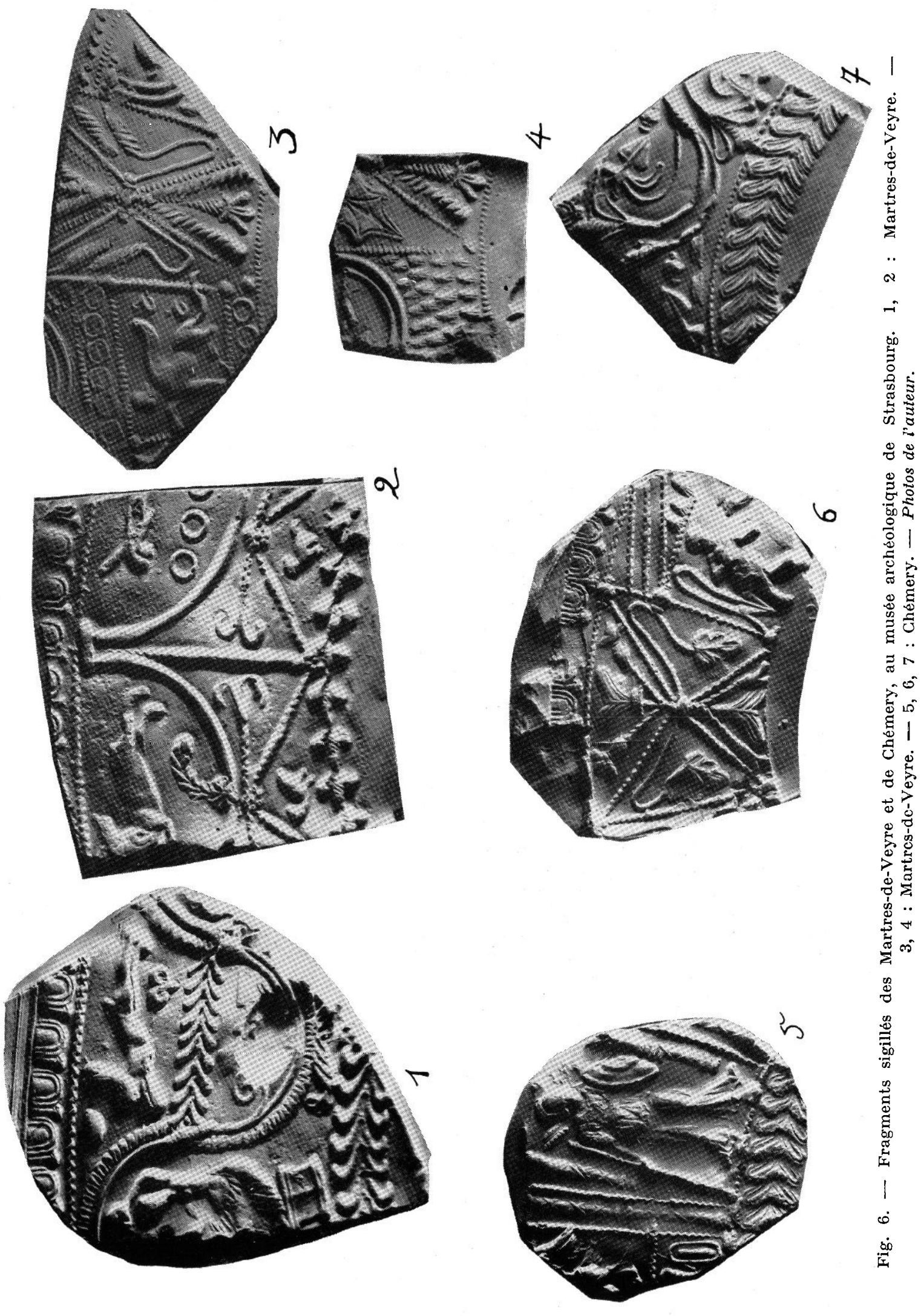


$* *$

La fin du $\mathrm{I}^{\mathrm{er}}$ siècle est une époque capitale pour l'évolution de la terre
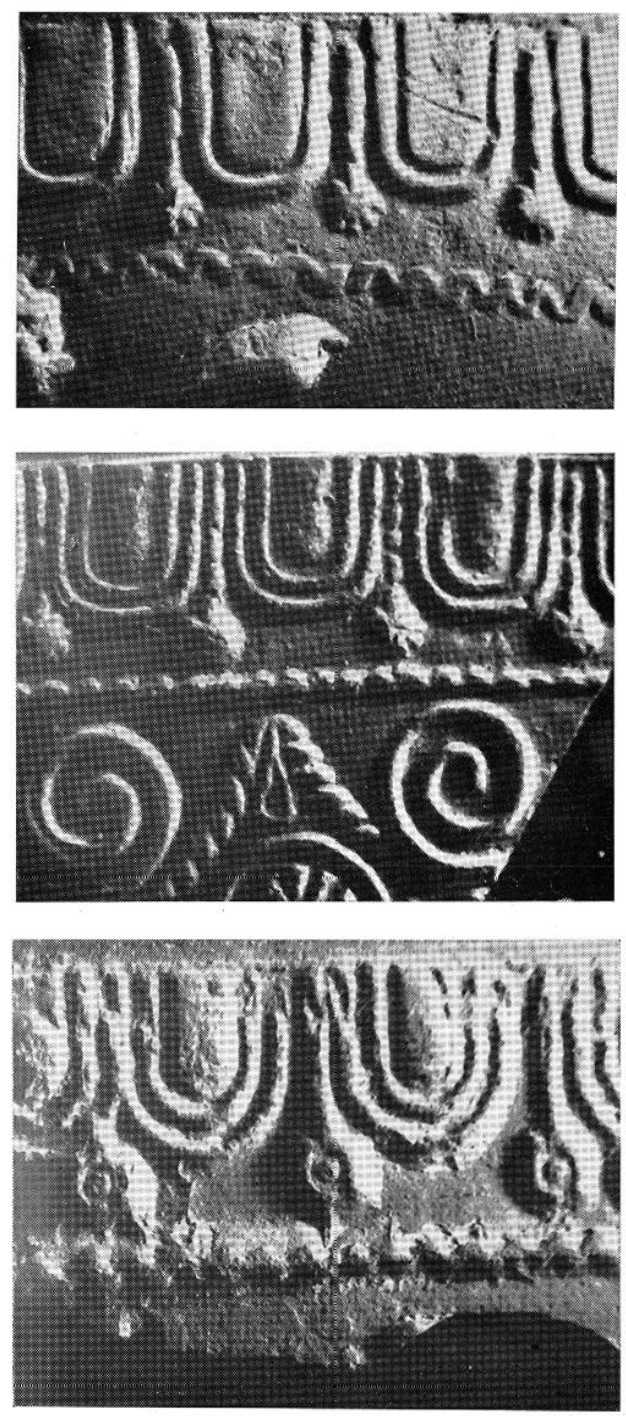

Fig. 7. - $a$ : Oves agrandies de Martres-deVeyre (Maitre aux amours, Maitre aux gladiateurs). - $b$ : oves agrandies de Chémery (second atelier, type no 1 de la fig. 4). - $-c$ : oves agrandies de Chémery (second atelier, type no 2 de la fig. 1). - Photos de l'auteur. sigillée gauloise. La décadence progressive et l'appauvrissement du Midi méditerranéen, l'activité et la prospérité grandissante de la région du Nord-Est de la Gaule ont eu pour conséquence des exodes d'industries, des fondations de filiales. La céramique sigillée décorée de reliefs constitue une documentation de choix, pour étudier ces phénomènes, non plus dans leur généralité, mais dans la précision bien circonscrite des cas individuels. Des rapprochements comme ceux qu'il convient de faire entre les Martres-de-Veyre et Chémery sont les seuls qui puissent permettre de déterminer le processus réel des faits.

L'officine des Martres-de-Veyre, à l'étude de laquelle R. Terrisse se consacre avec dévouement depuis de longues années, a été une rivale de celle de Lezoux. Elle semble avoir éclaté à la fin du $1^{\text {er }}$ siècle, sans doute précisément a cause de cette redoutable concurrence de Lezoux. Aussi est-ce principalement à elle, et non à Lezoux, qu'il convient d'attribuer la part prédominante des exodes de cadres de maîtrise et dans la formation des filiales. Nous trouvons dans toule la céramique du Nord-Est de la Gaule trop de motifs dérivés du répertoire fondamental des Martres-deVeyre, pour que cette influence n'ail pas été plus forte encore que nous ne le soupçonnons actuellement. On peut supposer qu'elle s'est exercée sur d'autres officines encore que celle de Chémery. Aussi conviendrait-il de diriger des recherches plus approfondies sur des officines [actuellement mal connues, comme celles de Mandeure, de Luxeuil, de la Madeleine et d'autres.

Jean-Jacques Hatr. 